\title{
Estimation of the second ventilatory threshold through ventricular repolarization profile analysis
}

\author{
Javier Milagro ${ }^{1,2}$ (다 \\ José A. Casajús ${ }^{3,5,6,7}$ \\ | Adrián Hernández-Vicente , $^{3,4}$
Nuria Garatachea $3,4,6,7,8$ \\ | David Hernando ${ }^{1,2}$ \\ ${ }^{1}$ Biomedical Signal Interpretation and Computational Simulation Group, Aragón
${ }^{2}$ CIBER in Bioengineering, Biomaterials and Nanomedicine, Madrid, Spain \\ ${ }^{3}$ GENUD (Growth, Exercise, Nutrition and Development) Research Group, Faculty of Health and Sport Sciences, University of Zaragoza, Zaragoza, Spain \\ ${ }^{4}$ Faculty of Health and Sport Science (FCSD), Department of Physiatry and Nursing, University of Zaragoza, Huesca, Spain \\ ${ }^{5}$ Faculty of Health Sciences (FCS), Department of Physiatry and Nursing, University of Zaragoza, Zaragoza, Spain \\ ${ }^{6}$ Agro-alimentary Institute of Aragón -IA2- (CITA-University of Zaragoza), Zaragoza, Spain \\ ${ }^{7}$ CIBER in Physiopathology of Obesity and Nutrition (CIBER-Obn), Madrid, Spain \\ ${ }^{8}$ Consejo Superior de Deportes, Madrid, Spain
}

\section{Correspondence}

Javier Milagro, Biomedical Signal

Interpretation and Computational

Simulation Group, Aragón Institute of

Engineering Research, University of

Zaragoza, C/ María de Luna 1, Edif. Ada

Byron, L.3.07 50018 Zaragoza, Spain.

Email: milagro@unizar.es

\section{Funding information}

H2020 European Research Council, Grant/ Award Number: ERC-2014-StG 638284;

Ministerio de Economía y Competitividad, Grant/Award Number: BES-2015-073694, PID2019-105674RB-I00 and RTI2018-

097723-B-I00; Ministerio de Educación,

Cultura y Deporte, Grant/Award Number:

FPU16/05879; Gobierno de Aragón, Grant/

Award Number: LMP124-18, LMP44_18

and T39-20R; Ministerio de Economía y

Competitividad; Ministerio de Ciencia e

Innovación; European Research Council,

Grant/Award Number: 638284,, T39-20R,

LMP124-18, FPU16 and 05879; Instituto de

Salud Carlos III; Ministerio de Educación,

Cultura y Deporte; CIBER-BBN; University of Zaragoza.

\begin{abstract}
Under the hypothesis that sympathetic control of ventricular repolarization may change once the second ventilatory threshold (VT2) has been reached, a novel methodology for non-invasive VT2 estimation based on the analysis of the T wave from the electrocardiogram (ECG) is proposed, and potential underlying physiological mechanisms are suggested. 25 volunteers $(33.4 \pm 5.2$ years $)$ underwent an incremental power cycle ergometer test $(25 \mathrm{~W} /$ minute). During the test, respiratory gas exchange and multi-lead ECG were acquired. The former was employed to determine VT2, used here as a reference, whereas the latter was used to compute the temporal profiles of an index of ventricular repolarization instability $(d \mathrm{~T})$ and its low-frequency (LF) oscillations ( $\mathrm{LF} d \mathrm{~T}$ ). The sudden increases observed in $d \mathrm{~T}$ and $\mathrm{LF} d \mathrm{~T}$ profiles above an established heart rate threshold were employed to derive $\mathrm{VT} 2$ estimates, referred to as $\mathrm{VT} 2_{d \mathrm{~T}}$ and $\mathrm{VT} 2_{\mathrm{LF} d \mathrm{~T}}$, respectively. Estimation errors of $-4.7 \pm 25.2 \mathrm{~W}$ were obtained when considering $\mathrm{VT} 2_{d \mathrm{~T}}$. Errors were lower than the one-minute power increment of $25 \mathrm{~W}$ in $68 \%$ of the subjects and lower than 50 $\mathrm{W}$ in $89.5 \%$ of them. When using $\mathrm{VT} 2_{\mathrm{LFdT}}$, estimation error was of $15.3 \pm 32.4 \mathrm{~W}$. Most of the subjects shared common characteristic $d \mathrm{~T}$ and LF $d \mathrm{~T}$ profiles, which could be reflecting changes in the autonomic control of ventricular repolarization before and after reaching VT2. The analysis of ventricular repolarization dynamics during exercise allows non-invasive ECG-based estimation of VT2, possibly in relation to changes in the autonomic control of ventricular electrical activity when VT2 is reached.
\end{abstract}




\section{K E Y W O R D S}

autonomic nervous system, exercise test, ventilatory threshold, ventricular repolarization

\section{1 | INTRODUCTION}

During "light-intensity" exercise, the aerobic energy production system combines blood $\mathrm{O}_{2}$ with carbohydrates and fats to synthesize adenosine triphosphate (ATP), which is the molecule providing the muscles with energy. However, the rhythm at which ATP is produced through the aerobic pathways turns out to be insufficient to maintain muscle activity at higher exercise intensity. As exercise intensity increases, the anaerobic glycolytic pathway is gradually favored. This route employs the glycogen stored in the muscles and releases lactate and $\mathrm{H}^{+}$ions as end products, this resulting in metabolic acidosis.

The $\mathrm{O}_{2}$ consumption above which anaerobic mechanisms are needed to complement aerobic energy production is referred to as aerobic threshold (AerT) and is linked with the first increase in blood lactate concentrations above resting values. ${ }^{2,3}$ During an exhaustive incremental test, when exercising at "moderate-intensity" (above the AerT), the bicarbonate buffer system manages the metabolic acidosis, resulting in hyperpnea elicited by the increase in the $\mathrm{CO}_{2}$. However, when exercising at "high-intensity," hyperpnea is not sufficient to eliminate the $\mathrm{CO}_{2}$ metabolic production, leading to an increase of ventilation until exhaustion, being this point called "threshold of decompensated metabolic acidosis" or anaerobic threshold (AnT), which slightly exceeds the maximal lactate steady state. ${ }^{3}$ It is acknowledged that the term "threshold" does not reflect the transitional nature of changes, and there is no complete absence of anaerobic metabolism even at rest, but this traditional terminology allows a didactic introduction without reducing the explanation to a single measurement method, for example, gas exchange.

Apart from representing an inflection point in the way the body obtains energy to maintain its exercise capacity, AnT is also regarded as the frontier beyond which the cardiovascular system limits the endurance capacity. In this regard, accurate estimation of AnT is of major interest in the field of sport sciences, as it could be used to design better training routines, quantify athletes' performance or prevent them from overtraining. Moreover, it has clinical value in the assessment of exercise responses involving the pulmonary, cardiovascular, and skeletal muscle systems, as well as in risk stratification. ${ }^{4}$

Different methodologies for estimation of AnT based on the analysis of blood lactate (lactate threshold, $\mathrm{AnT}_{\mathrm{LA}}$ ) and gas exchange (second ventilatory threshold, VT2) ${ }^{5}$ have been proposed in the literature. However, whereas the former requires repetitive acquisition of blood samples, the latter employs expensive devices, so that its use is limited to laboratories and specialized centers. For these reasons, there is a growing interest in non-invasive estimation of AnT using a variety of approaches, such as the analysis of electromyographic (EMG) activity ${ }^{6,7}$ or electrocardiogram (ECG)-derived indices like heart rate $(\mathrm{HR})^{8}$ or heart rate variability (HRV) ${ }^{9,10}$ A limitation of EMG-based approaches is that the use of wearable devices is not as extended as in the case of cardiac activity assessment. On the other hand, approaches assessing autonomic nervous system activity by HRV measurements are compromised during high-intensity exercise, since the mechanic effect of respiration has been acknowledged as the main modulator of HR in this condition. ${ }^{11}$ Hence, HRV might not represent an appropriate approach for studying the role of the autonomic nervous system in the transition to anaerobic metabolism during high-intensity exercise. In a recent work, ${ }^{12}$ a methodology for estimation of $\mathrm{AnT}_{\mathrm{LA}}$ was proposed based on the analysis of an index of ventricular repolarization instability, $d \mathrm{~T}$, and its characteristic temporal pattern in relation to HR. The use of blood lactate levels as a gold standard for detecting AnT in that work may, however, result in high uncertainty, considering that it can be only assessed on a discrete-time basis, usually with large sampling periods.

In this study, a novel method for AnT estimation from the analysis of ventricular repolarization dynamics is proposed and its performance assessed using VT2 as a reference, provided that it can be evaluated on a more continuous-time basis than lactate levels. Our proposal departs from recent published works where low-frequency (LF) oscillations of $d \mathrm{~T}$ have been shown to represent sympathetic modulation of ventricular repolarization. ${ }^{13,14}$ Under the hypothesis that VT2 may be related to an increase in sympathetic activity due to the need of faster ventilation, this study explores VT2 estimation from $d \mathrm{~T}$ and from its LF oscillations (LF $d \mathrm{~T}$ ). Additionally, physiological mechanisms possibly underlying $d \mathrm{~T}$ and $\mathrm{LF} d \mathrm{~T}$ temporal profiles during the exercise test are proposed.

\section{$2 \mid$ METHODS}

\subsection{Dataset}

Twenty-five healthy male volunteers $(33.4 \pm 5.2$ years $)$, whose descriptive characteristics are summarized in Table 1, were recruited. All of them practiced aerobic training a minimum of 3 times/week were not active smokers and did not report any respiratory or cardiac disorder by the time of data 
TA B L E 1 Demographics of the participants (mean \pm standard deviation)

\begin{tabular}{lr}
$\mathbf{N}$ & \multicolumn{1}{c}{$\mathbf{2 5}$} \\
\hline Age (years) & $33.4 \pm 5.2$ \\
Height $(\mathrm{cm})$ & $178.0 \pm 5.5$ \\
Weight $(\mathrm{kg})$ & $74.8 \pm 7.0$ \\
BMI $\left(\mathrm{kg} / \mathrm{m}^{2}\right)$ & $23.6 \pm 2.1$ \\
\hline
\end{tabular}

Abbreviation: BMI, body mass index.

acquisition. The volunteers performed a submaximal cycle ergometer test (VIAsprint 150 P, Ergoline, Germany), divided in three stages. In the first stage, subjects remained seated and without talking for 5 minutes. Afterward, they performed a cycle ergometer test, with an initial workload of $75 \mathrm{~W}$ that was increased by $25 \mathrm{~W}$ every minute. The cadence frequency was fixed at $80 \mathrm{rpm}$, and the workload kept on increasing until the volunteers reached $90 \%$ of their maximum HR (the maximum HR was determined as in15), following which the workload was kept fixed for 2 more minutes. Finally, subjects underwent a recovery stage, in which they were asked to keep on pedaling at $75 \mathrm{~W}$ for 3 to 5 more minutes. All the volunteers were requested to avoid food, tobacco, alcohol, or caffeine in the 3 hours prior to the test, to avoid heavy physical activity on the day of the test and to drink plenty of fluids during the previous 24 hours.

During the test, a high-resolution multi-lead ECG was acquired using a Holter device (Mortara 48-hour H12+, Mortara Instrument, Milwaukee, Wisconsin). Leads I, II, III, aVL, aVR, aVF, V4, V5, and V6 were acquired at $1000 \mathrm{~Hz}$. On the other hand, $\mathrm{O}_{2}$ and $\mathrm{CO}_{2}$ consumption $\left(\dot{\mathrm{V}}_{\mathrm{O}_{2}}\right.$ and $\dot{\mathrm{V}}_{\mathrm{CO}_{2}}$, respectively) and minute ventilation $\left(\dot{\mathrm{V}}_{\mathrm{E}}\right)$ were assessed with an Oxycon Pro device (Jaeger/Viasys, Germany). The protocol was approved by the Ethics Committee of Aragón in accordance with the Declaration of Helsinki and written informed consent was obtained from all subjects.

\section{2 | Determination of the anaerobic threshold (AnT)}

The second ventilatory threshold (VT2) was determined by an expert in sport physiology from the ventilatory equivalents of $\mathrm{O}_{2}$ and $\mathrm{CO}_{2}\left(\dot{\mathrm{V}}_{\mathrm{E}} / \dot{\mathrm{V}}_{\mathrm{O}_{2}}\right.$ and $\dot{\mathrm{V}}_{\mathrm{E}} / \dot{\mathrm{V}}_{\mathrm{CO}_{2}}$, respectively) of each subject. Specifically, the moment at which there was a concomitant rapid increase in $\dot{\mathrm{V}}_{\mathrm{E}} / \dot{\mathrm{V}}_{\mathrm{O}_{2}}$ and $\dot{\mathrm{V}}_{\mathrm{E}} / \dot{\mathrm{V}}_{\mathrm{CO}_{2}}$ was set as VT2. ${ }^{2}$ The determined VT2 annotations were used as the reference anaerobic gas exchange threshold. The AerT, that is, first ventilatory threshold (VT1) calculation is beyond the scope of this paper, but it is understood as the first rise in $\dot{\mathrm{V}}_{\mathrm{E}} / \dot{\mathrm{V}}_{\mathrm{O}_{2}}$ without a parallel increase in $\dot{\mathrm{V}}_{\mathrm{E}} / \dot{\mathrm{V}}_{\mathrm{O}_{2}}$ and $\dot{\mathrm{V}}_{\mathrm{E}} / \dot{\mathrm{V}}_{\mathrm{CO}_{2}}$
.$^{2}$ Following the conceptual framework described by Meyer et al $^{3}$ : "light-intensity" (<VT1), "moderate-intensity" (VT1 to VT2), and "high-intensity" ( $>$ VT2) will be used henceforth as exercise intensity domain descriptors. ${ }^{16}$

\subsection{Repolarization dynamics}

Baseline wander, calculated by using a forward-backward low-pass filter with $0.5 \mathrm{~Hz}$ cut-off frequency, was subtracted from the ECG recordings. Beat detection and delineation were performed by using an automatic wavelet-based approach proposed by Martínez et al, ${ }^{17}$ which rendered temporal marks for the onset and end of the $\mathrm{T}$ waves $\left(\mathrm{T}_{\text {on }}\right.$ and $\mathrm{T}_{\text {off }}$, respectively). Since there were not three orthogonal leads available and the absence of V2 did not allow to calculate the Frank's lead configuration, the three most orthogonal leads, namely V4, V6, and aVF, were considered. After low-pass filtering the ECG signals to remove high-frequency noise (forward-backward filter with $30 \mathrm{~Hz}$ cut-off frequency), the $\mathrm{T}$ waves were extracted from each lead based on $\mathrm{T}_{\text {on }}$ and $\mathrm{T}_{\text {off }}$ delineation marks. The repolarization dynamics profile, $d \mathrm{~T}$, was constructed as follows. First, for each beat $i$, the T waves of the 3 employed leads were forced to have a common origin at $0 \mu \mathrm{V}$ by subtracting from them the amplitude of their respective first samples. Next, the length of the $T$ waves was truncated to the point when one of them returned to zero to avoid negative values. Finally, the three-dimensional repolarization vector corresponding to beat $i$ was constructed by considering the mean amplitude of the three $\mathrm{T}$ waves as its coordinates. The value of $d \mathrm{~T}(i)$ was computed as the angular difference between the repolarization vectors of beats $i$ and $i-1$. An example of this process is displayed in Figure 1. To improve the robustness in the computation of $d \mathrm{~T}(i)$, this was only calculated when the $\mathrm{T}$ waves corresponding to beats $i$ and $i-1$ presented a Pearson correlation coefficient $\geq 0.9$ in all the employed leads.

On top of the above described three-dimensional approach applied onto ECG leads V4, V6, and aVF, a two-dimensional approach was additionally developed based on the fact that visual analysis of the ECG signals revealed that lead aVF was noisy in approximately half of the recordings during the moderate-to-high intensity exercise. Based on that, in our two-dimensional approach, $d \mathrm{~T}$ was estimated from leads $\mathrm{V} 4$ and V6 only, with the same criterion on the correlation coefficient $\geq 0.9$ being applied. The next steps in the analysis, described below, were identical for the three- and two-dimensional approaches.

Also, the instantaneous HR was computed from the time difference between consecutive beats. The HR and $d \mathrm{~T}$ signals were interpolated to $4 \mathrm{~Hz}$ and low-pass filtered (forward-backward filter with $0.01 \mathrm{~Hz}$ cut-off frequency) to obtain the HR and $d \mathrm{~T}$ profiles (see Figure 2A). 

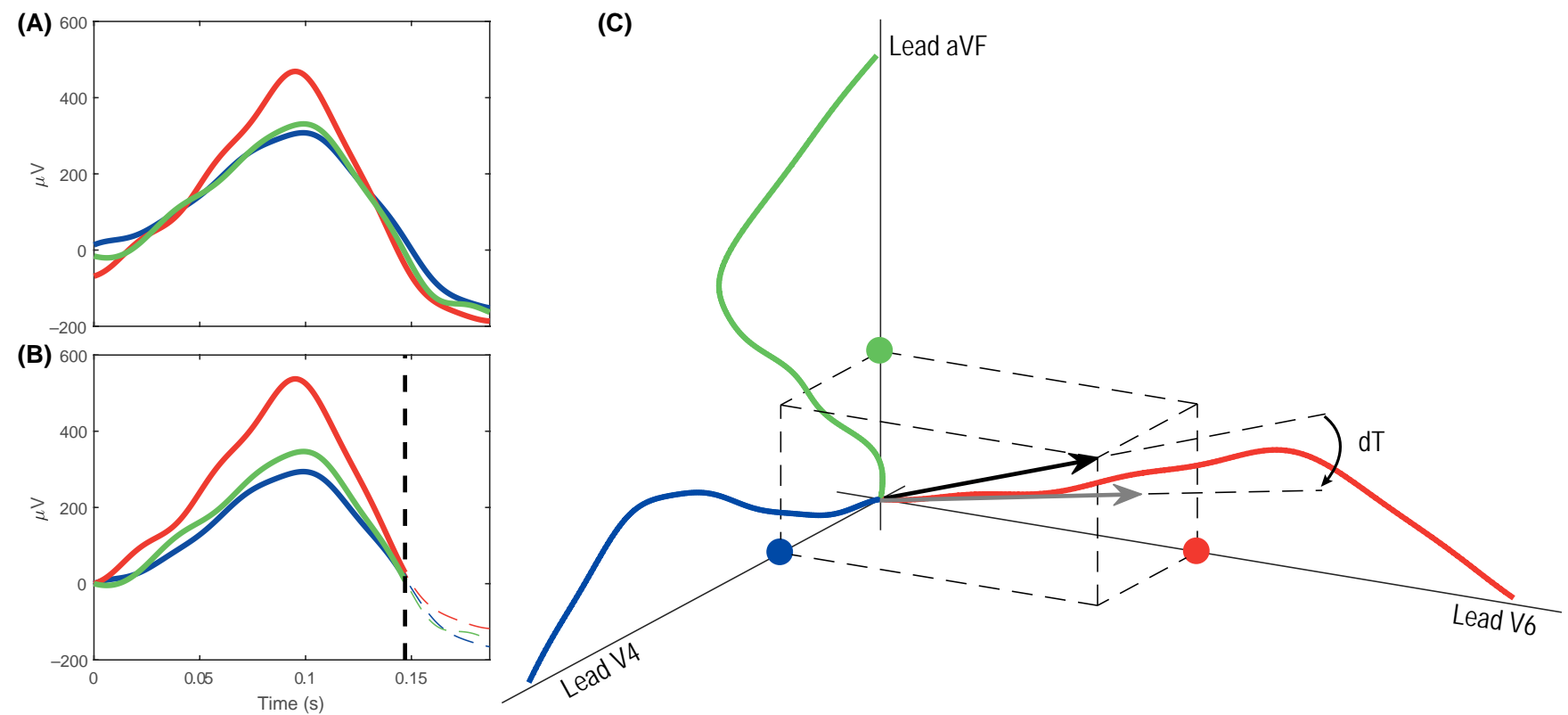

F I G URE 1 Calculation of the $d$ T series. In A, the original T waves of the three considered leads (V4: blue, V6: red, aVF: green) are displayed. In B, the value of the first sample was subtracted from each $\mathrm{T}$ wave so as to make them have a common origin and, additionally, they were truncated to the first zero-crossing to end at the same value, which is marked with a black dashed line in the graphic. Only the portion of the $\mathrm{T}$ waves displayed in bold was used to compute $d \mathrm{~T}$. In $\mathrm{C}$, the $\mathrm{T}$ waves are displayed along three axes representative of leads V4, V6, and aVF. The values of their mean amplitudes (displayed with filled circles) were used to construct the repolarization vector (black arrow). Afterward, $d \mathrm{~T}$ was calculated as the angular difference between this vector and the vector corresponding to the previous beat (gray arrow)

LF oscillations of $d \mathrm{~T}$ were additionally assessed. For this purpose, the $d T$ profile (Figure 2A), thick green line) was subtracted from the interpolated $d \mathrm{~T}$ series (Figure 2A), thin green line), and the short-term Fourier transform (STFT) of the detrended $d \mathrm{~T}$ series was computed (120-second Blackman window, slid sample-by-sample). Next, the $\mathrm{LF} d \mathrm{~T}$ series was calculated as the total power within the $[0.04,0.15] \mathrm{Hz}$ band at each time instant. The resulting series was low-pass filtered, in the same way as for the HR and $d \mathrm{~T}$ series, to obtain the $\mathrm{LF} d \mathrm{~T}$ profile. An example of the $\mathrm{LF} d \mathrm{~T}$ profile of one subject is displayed in Figure $2 \mathrm{~B}$ ), whereas the corresponding time-frequency distribution is depicted in Figure 2D). To analyze the effect of the cardiolocomotor coupling in the computation of $\mathrm{LF} d \mathrm{~T}$, the frequency alias corresponding to $\mathrm{HR}(t)-\mathrm{F}_{\mathrm{C}}$ and $\left|2 \mathrm{~F}_{\mathrm{c}}-\mathrm{HR}(\mathrm{t})\right|$ was studied as proposed in, ${ }^{18}$ where $\mathrm{HR}(t)$ represents the instantaneous HR and $\mathrm{F}_{\mathrm{C}}$ accounts for the pedaling cadence. An example showing the temporal evolution of these components is displayed in Figure $2 \mathrm{D})$. The study of the frequency alias due to cardiolocomotor coupling is fundamental to assess whether changes in $\mathrm{LF} d \mathrm{~T}$ are indeed due to variations in autonomic modulation of cardiac electrical activity or to the mechanical effect of pedaling.

\subsection{Second ventilatory threshold estimation}

The analysis of the $d \mathrm{~T}$ profiles revealed a characteristic sudden increase in the repolarization instability in the vicinity of VT2 (see Figure 2A)). The time instant at which the fastest variation in $d \mathrm{~T}$ occurred was located by identifying the time associated with the maximum of its first derivative. The point at which this fast increase in $d \mathrm{~T}$ started was located as the local minimum preceding the maximum of its first derivative, and this point was selected as an estimate of VT2, being denoted as VT2 ${ }_{d \mathrm{~T}}$. An example showing VT2 estimation from the derivative of the $d \mathrm{~T}$ profile is depicted in Figure 2A). In some subjects, a local rather than a global maximum of the derivative of $d \mathrm{~T}$ was found late during the exercise test, with the absolute maximum occurring close to the onset of exercise. For this reason, a lower threshold in HR was established, below which a switch toward the anaerobic pathways should not be expected. This predefined HR threshold was set at $77 \%$ of the maximum HR of each subject based on a previous study by Goldberg et $\mathrm{al}^{19}$ reporting that this value was always below the HR measured at VT1, independently of the physical condition. Moreover, it is in accordance with studies reporting a HR of $90 \%$ of the maximum HR at the training intensity of AnT (assessed either from $\mathrm{AnT}_{\mathrm{LA}}$ or VT2). ${ }^{20,21}$

Additionally, an estimate of VT2 from the LF $d$ T profile was obtained. The same criteria as those described for the $d \mathrm{~T}$ profile were employed. An example of the VT2 estimate from the first derivative of the $\mathrm{LF} d \mathrm{~T}$ series is displayed in Figure 2B). In this case, the resulting estimate is referred to as $\mathrm{VT} 2_{\mathrm{LF} d \mathrm{~T}}$. 


\subsection{Heart rate variability assessment}

HRV was assessed to aid in the interpretation of the results. For this purpose, we estimated the modulating signal (which is a representation of the autonomic modulation of HR) using the time-varying integral pulse frequency modulation model that accounts for the variations in mean $\mathrm{HR} .{ }^{22}$ After resampling the series at $4 \mathrm{~Hz}$, we computed its STFT following the same methods described in section 2.3. The instantaneous LF power $\left(\mathrm{P}_{\mathrm{LF}}\right.$, related to both sympathetic and parasympathetic modulation of HR) was obtained as the power content within the $[0.04,0.15] \mathrm{Hz}$ band. ${ }^{23}$ On the other hand, the high-frequency

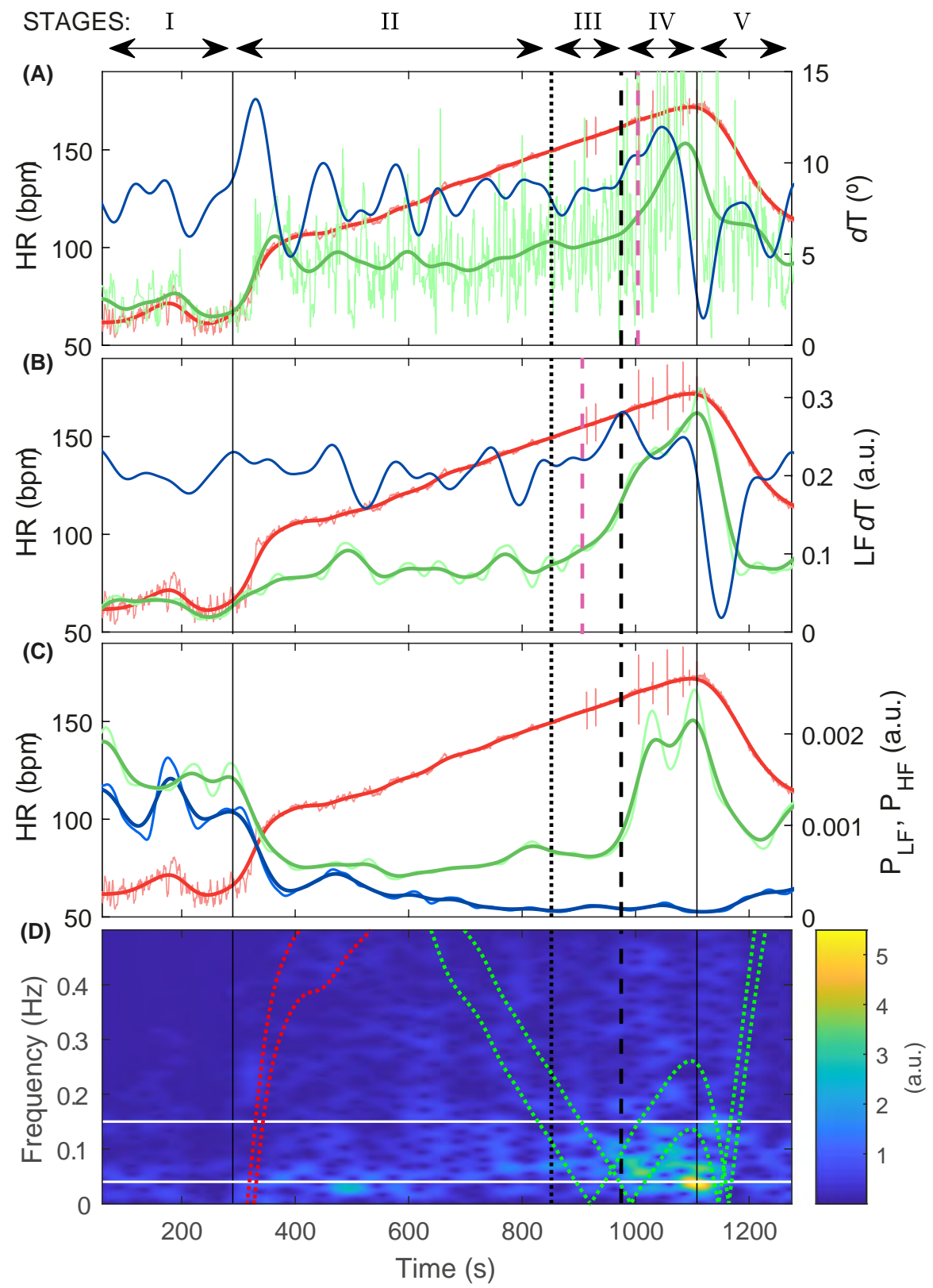

F I G URE 2 A, Instantaneous HR (red, left axis) and $d$ T (green, right axis) during the exercise test for a given subject. The HR and $d$ T profiles are represented in bold. Moreover, the first derivative of the $d \mathrm{~T}$ profile is displayed (blue, rescaled for representation purposes). B, Temporal evolution of the LF $d \mathrm{~T}$ (green, right axis), with its profile represented in bold and its first derivative in blue (rescaled for representation purposes). C, Temporal evolution of the LF (blue) and HF (green) components of HRV. D, Time-frequency distribution of $d \mathrm{~T}$ (detrended). The limits of the band used for computing LF $d$ T are shown in white, whereas the dotted red and green lines represent the limits of a $0.125 \mathrm{~Hz}$ band centered in the alias terms $\mathrm{HR}(t)-\mathrm{Fc}$ and $|2 \mathrm{Fc}-\mathrm{HR}(\mathrm{t})|$, respectively $\left(\mathrm{HR}(t)\right.$ represents the instantaneous heart rate, whereas $\mathrm{F}_{\mathrm{c}}$ accounts for the pedaling cadence). The dotted black lines account for the position when sympathetic activity (as reflected by ventricular repolarization) starts to increase, whereas the dashed black and pink lines represent the occurrence time of the reference and estimated VT2, respectively. Five different stages are distinguished during the exercise test (delimited by the black solid, dotted, and dashed lines), as indicated above the upper panel (see text for details) 
(HF) power $\left(\mathrm{P}_{\mathrm{HF}}\right)$ was computed using a time-varying band, specifically as the power content between $0.15 \mathrm{~Hz}$ and half the instantaneous HR (in Hz), since HR represents the intrinsic sampling rate of HRV. ${ }^{24}$ Whereas $\mathrm{P}_{\mathrm{HF}}$ is related to vagal HR modulation in basal conditions, ${ }^{23}$ it has been suggested to represent the mechanical modulation of HR due to breathing during high-intensity exercise. ${ }^{11}$ An example of the evolution of $\mathrm{P}_{\mathrm{LF}}$ and $\mathrm{P}_{\mathrm{HF}}$ during the exercise test for a given subject is displayed in Figure 2(C). Please note that arbitrary units were used for LF and HF powers, as these were obtained from the time-varying integral pulse frequency modulation model.

\section{6 | Performance evaluation}

The performance of the two proposed methodologies for VT2 estimation was assessed by calculating the estimation error as the difference with respect to the annotated VT2 time occurrence. Normality of the residuals was checked using a Kolmogorov-Smirnov test. Bland-Altman plots corresponding to VT2 estimation using the $d \mathrm{~T}$ and $\mathrm{LF} d \mathrm{~T}$ profiles were computed. All the results are presented in terms of workload, so as to facilitate comparison to previous studies. The VT2 estimates were converted from seconds to Watts by assuming a linear increment in the workload. Since it is difficult to establish a priori boundaries for acceptable bias and precision, we took previous works as a reference. In this way, we established a priori precision of the Bland-Altman plot (ie, limits of agreement) of $\pm 50 \mathrm{~W}$, which is approximately the value obtained in. ${ }^{12}$ With respect, to the bias, it should be kept within the limits of inter-evaluator error in the visual estimation of the ventilatory threshold, which has been reported to lie within 3 to $16 \%$ of $\dot{\mathrm{V}}_{\mathrm{O}_{2, \max }}{ }^{25}$

Since $d \mathrm{~T}$ was computed by using both two and three leads, differences in the performance of the two-dimensional VT2 estimation approach with respect to the three-dimensional approach were evaluated. If the condition that consecutive $\mathrm{T}$ waves in a given lead must show a correlation larger than 0.9 was not satisfied for 100 consecutive beats, the corresponding recording was not included in the analysis. The threshold for the correlation coefficient was empirically set at 0.9 to include at least $80 \%$ of the $\mathrm{T}$ waves when using thee two-dimensional approach for $d \mathrm{~T}$ computation.

\section{3 | RESULTS}

From the 25 volunteers enrolled in the study, 5 were discarded because of misleading $\mathrm{T}$ wave delineation during the moderate-to-high intensity exercise due to noisy ECGs (more than 100 consecutive beats without a correlation index $\geq 0.9$ among consecutive $\mathrm{T}$ waves). Another subject was not included due to missing respiratory gas exchange information.
TA B LE 2 Results of the exercise test (mean \pm standard deviation)

\begin{tabular}{|c|c|}
\hline max. HR (bpm) & $179.0 \pm 8.4$ \\
\hline$\% \max . \mathrm{HR}$ at VT2 & $87.8 \pm 4.2$ \\
\hline$\% \max . \mathrm{HR}$ at $\mathrm{VT} 2_{d \mathrm{~T}}$ & $88.5 \pm 3.5$ \\
\hline$\% \max . \mathrm{HR}$ at $\mathrm{VT} 2_{\mathrm{LF} d \mathrm{~T}}$ & $86.2 \pm 4.8$ \\
\hline$\dot{\mathrm{V}}_{\mathrm{O}_{2, \max }}(1 / \min )$ & $4.3 \pm 0.4$ \\
\hline$\dot{\mathrm{V}}_{\mathrm{O}_{2}}$ at $\mathrm{VT} 2(\mathrm{~L} / \mathrm{min})$ & $3.4 \pm 0.5$ \\
\hline$\% \dot{\mathrm{V}}_{\mathrm{O}_{2, \max }}$ at $\mathrm{VT} 2$ & $79.8 \pm 10.5$ \\
\hline$\dot{\mathrm{V}}_{\mathrm{O}_{2}}$ at $\mathrm{VT} 2_{d \mathrm{~T}}(\mathrm{~L} / \mathrm{min})$ & $3.5 \pm 0.4$ \\
\hline$\% \dot{\mathrm{V}}_{\mathrm{O}_{2, \max }}$ at $\mathrm{VT} 2_{d \mathrm{~T}}$ & $80.8 \pm 8.6$ \\
\hline$\dot{\mathrm{V}}_{\mathrm{O}_{2}}$ at $\mathrm{VT} 2_{\mathrm{LF} d \mathrm{~T}}(1 / \mathrm{min})$ & $3.3 \pm 0.4$ \\
\hline$\% \dot{\mathrm{V}}_{\mathrm{O}_{2, \max }}$ at $\mathrm{VT} 2_{\mathrm{LF} d \mathrm{~T}}$ & $76.7 \pm 11.5$ \\
\hline max. workload (W) & $359.2 \pm 47.3$ \\
\hline$\%$ max. workload at VT2 & $84.6 \pm 6.9$ \\
\hline$\%$ max. workload at VT2 $2_{d \mathrm{~T}}$ & $86.8 \pm 5.4$ \\
\hline$\%$ max. workload at $\mathrm{VT} 2_{\mathrm{LF} d \mathrm{~T}}$ & $81.7 \pm 7.9$ \\
\hline
\end{tabular}

Max. HR refers to the maximum HR achieves during the test.

Abbreviations: VT2, second ventilatory threshold; VT2 $2_{d \mathrm{~T}}$, second ventilatory threshold estimated from ventricular repolarization instability; VT2 $\mathrm{LF}_{d \mathrm{~T}}$, second ventilatory threshold estimated from the low-frequency oscillations of ventricular repolarization instability; $\dot{\mathrm{V}}_{\mathrm{O}_{2}}: \mathrm{O}_{2}$ consumption, $\dot{\mathrm{V}}_{\mathrm{O}_{2, \max }}$ : maximum $\mathrm{O}_{2}$ consumption during the test.

Estimation results are summarized in Table 2, whereas Bland-Altman plots to assess VT2 estimation when using $d \mathrm{~T}$ and LFdT profiles are displayed in Figure 3, in all cases using the two-lead approach. As shown in Figure 3, there was low dispersion in the estimation errors around the mean value, especially in the case of $\mathrm{VT} 2_{d \mathrm{~T}}$.

When using $d \mathrm{~T}$ to estimate VT2, a mean error of $-4.7 \pm$ $25.2 \mathrm{~W}$ was measured, this being $15.3 \pm 32.4 \mathrm{~W}$ when employing the LFdT series. Since the workload was increased by $25 \mathrm{~W}$ per minute, the error in the estimation by VT $2_{d \mathrm{~T}}$ was lower than 1 minute for most of the subjects $(68 \%)$ and lower than 2 minutes for almost all of them (89.5\%), whereas it was generally kept below 2 minutes in the case of $\mathrm{VT} 2_{\mathrm{LF} d \mathrm{~T}}$ (78.9\% of subjects). The absolute workload values at VT2, $\mathrm{VT} 2_{d \mathrm{~T}}$, and $\mathrm{VT} 2_{\mathrm{LF} d \mathrm{~T}}$ are displayed in Table 2 . In terms of $\dot{\mathrm{V}}_{\mathrm{O}_{2}}$ , the mean error when using $d \mathrm{~T}$ was of $-0.04 \pm 0.23 \mathrm{~L} / \mathrm{min}$ $\left(-1.02 \pm 5.83 \%\right.$ of $\left.\dot{\mathrm{V}}_{\mathrm{O}_{2, \max }}\right)$, whereas it increased to $0.15 \pm$ $0.32 \mathrm{~L} / \min \left(3.08 \pm 7.34 \%\right.$ of $\left.\dot{\mathrm{V}}_{\mathrm{O}_{2, \max }}\right)$ when using LFdT.

Regarding the $d \mathrm{~T}$ profile, the dynamics shown in Figure 2A) were shared by the majority of the volunteers, with a sudden increase above the resting level in response to the exercise onset, followed by a plateau-like period and a new increase occurring in the vicinity of VT2. Finally, there was a decrease in $d \mathrm{~T}$ corresponding to the recovery stage. This behavior was very similar in the case of LFdT (Figure 2B). To assess whether the increase in LFdT in relation to VT2 could be due to the presence of harmonic components, the 

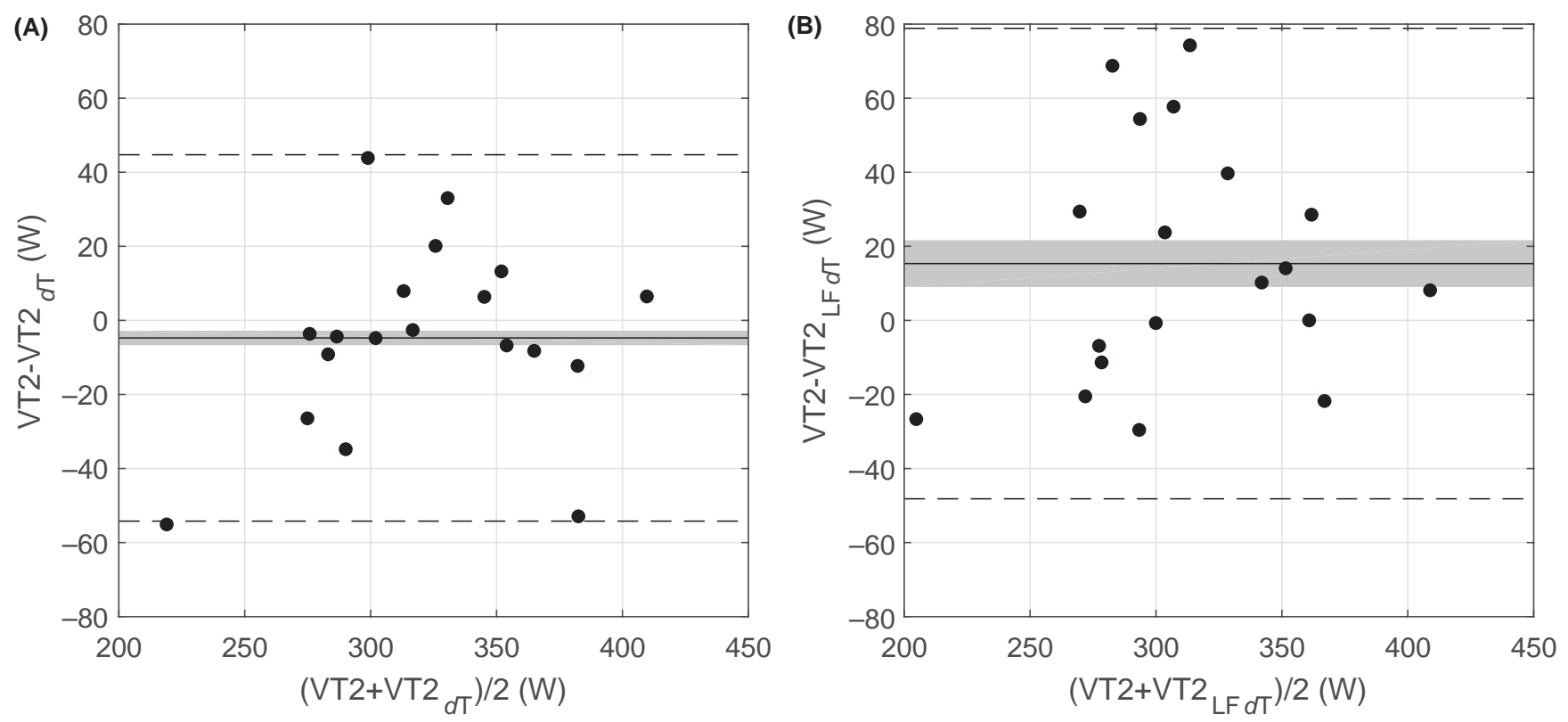

F I G U RE 3 Bland-Altman plots (difference vs mean) of the errors in the estimation of the VT2 using $d$ T (a) or LFdT (b) series. The solid line represents the mean of the estimation error, whereas the dashed lines account for the confidence intervals (mean \pm 1.96 standard deviations). The filled circles represent the values for the different subjects. The shaded area represents the confidence interval for the means of the estimation error

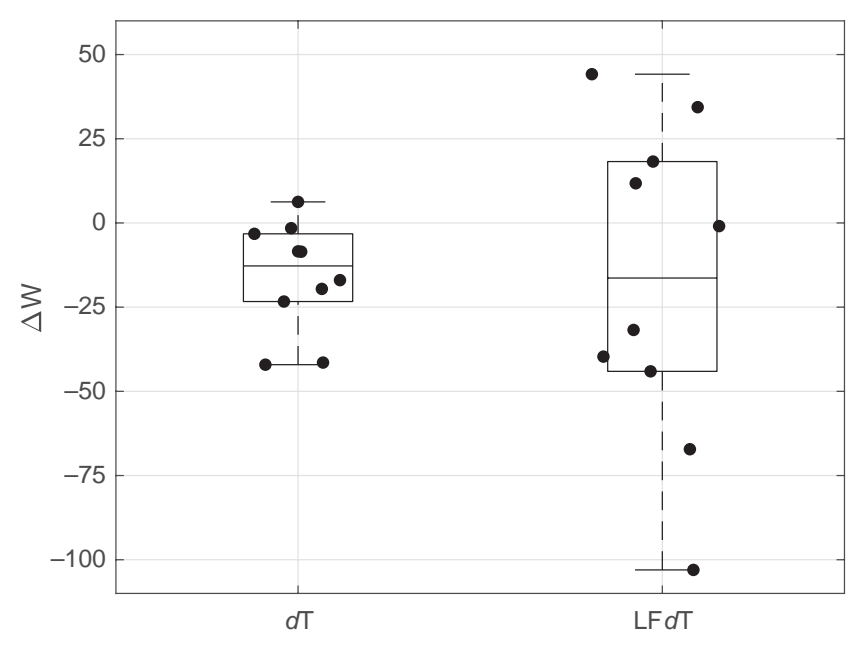

F I G URE 4 Difference in the VT2 (W) estimation when using three vs two leads, considering the $d \mathrm{~T}$ and the $\mathrm{LF} d \mathrm{~T}$ series. The filled circles represent the values for the different subjects

alias caused by the cardiolocomotor coupling were analyzed, as displayed in Figure 2D). We did not observe frequency components within these bands in a consistent manner. To assess the relationship between the $d \mathrm{~T}$ or $\mathrm{LF} d \mathrm{~T}$ profiles and $\mathrm{HR}$, we computed their inter-subject mean Spearman correlation coefficients, which were $0.57 \pm 0.24$ and $0.44 \pm 0.29$, respectively. We also considered the Spearman correlation between the $d \mathrm{~T}$ or LF $d \mathrm{~T}$ profiles and HRV measurements. In the case of $d \mathrm{~T}$, correlation with $\mathrm{P}_{\mathrm{LF}}$ and $\mathrm{P}_{\mathrm{HF}}$ was of -0.45 \pm 0.26 and $-0.06 \pm 0.43$, respectively, whereas these values were of $-0.35 \pm 0.28$ and $0.01 \pm 0.39$ for $\mathrm{LF} d \mathrm{~T}$.
Next, the results obtained by using three leads are presented for the 10 recordings that could be analyzed. Differences in the estimation performance between the two- and the three-lead approaches are displayed in Figure 4 (results for three leads minus results for two leads). For the three-lead approach, the mean estimation error in terms of $\dot{\mathrm{V}}_{\mathrm{O}_{2}}$ when using $d \mathrm{~T}$ was of $0.15 \pm 0.32 \mathrm{~L} / \min (3.24 \pm 7.82 \%$ of $\left.\dot{\mathrm{V}}_{\mathrm{O}_{2, \max }}\right)$, whereas it increased to $0.26 \pm 0.38(5.94 \pm 8.64 \%$ of $\dot{\mathrm{V}}_{\mathrm{O}_{2, \max }}$ ) when using LF $d \mathrm{~T}$. For the three-lead approach, estimation errors were below 1 minute for $60 \%$ and $50 \%$ of subjects, when considering VT2 $2_{d \mathrm{~T}}$ or VT2 $2_{\mathrm{LF} d \mathrm{~T}}$, respectively.

\section{4 | DISCUSSION}

In this work, VT2 was estimated during an incremental cycle ergometer test through the analysis of ventricular repolarization dynamics. The temporal profiles of an index of repolarization instability, $d \mathrm{~T}$, and of its LF oscillations, $\mathrm{LF} d \mathrm{~T}$, showed sudden increases after some minutes of exercise, once an established threshold on HR had been reached. The time point at which a change in the tendency of the overall repolarization dynamics (or its low-frequency content) occurred was identified and used to define estimates of VT2. The proposed methods yielded estimation errors lower than $50 \mathrm{~W}$, corresponding to 2 minutes of the exercise test, for the vast majority of subjects and lower than 1 minute for many of them. These results improve those of previous works in which, although the reported errors were likewise lower than $50 \mathrm{~W}$, the translation from 
Watts to minutes resulted in larger errors due to the nature of the exercise test. ${ }^{12}$ When evaluated in terms of $\dot{\mathrm{V}}_{\mathrm{O}_{2}}$, the mean estimation error was of $1 \%$ and $3 \%$ for $d \mathrm{~T}$ and $\mathrm{LF} d \mathrm{~T}$, respectively, being these errors lower than the inter-evaluator agreement in the visual determination reported in, ${ }^{25}$ which was comprised within 3\%-16\%.

The good performance achieved by both $\mathrm{VT} 22_{d \mathrm{~T}}$ and VT2 $2_{\mathrm{LF} d \mathrm{~T}}$ suggests that these two methods, particularly the first one, could be suitable for non-invasive VT2 estimation.

Our proposed methods are based on the hypothesis that, upon reaching VT2, there is a surge in sympathetic activity due to the need of faster ventilation, this resulting in alterations in sympathetically modulated ventricular repolarization. Several works have addressed the study of ventricular repolarization dynamics and its autonomic regulation, aiming both at providing improved markers for its quantification and at shedding light on its underlying physiological mechanisms. Rizas et $\mathrm{al}^{13}$ proposed the calculation of the angle $d \mathrm{~T}$ between successive repolarization vectors to subsequently evaluate the magnitude of its LF oscillations by the periodic repolarization dynamics (PRD) index. PRD has been suggested as a non-invasive measurement of the effect of sympathetic activity on ventricular myocardial repolarization on the basis of the observed increase in PRD following sympathetic activation and decrease in PRD after adrenergic blockade. ${ }^{13,14}$ Other studies have also investigated LF oscillations of repolarization dynamics at the level of ventricular epicardial electrograms and cellular action potentials, both in vivo ${ }^{26,27}$ and in silico, ${ }^{28,29}$ confirming the presence of an enhanced LF oscillatory pattern in ventricular repolarization in response to sympathetic provocation and ascertaining potential ionic mechanisms behind it.

Nevertheless, ventricular repolarization could be altered by several other causes, such as an increase in HR or mechanical effects. Hamm et $a l^{12}$ conducted an electrophysiological study in which they induced fixed atrial pacing with an incremental increase in HR, observing no variation in repolarization instability measured from the $d \mathrm{~T}$ angles with respect to the non-paced condition. To assess the relationship between HR, HRV, and repolarization instability in our study, we computed the average Spearman correlation between each of the HR, $\mathrm{P}_{\mathrm{LF}}$, and $\mathrm{P}_{\mathrm{HF}}$ profiles with the $d \mathrm{~T}$ and $\mathrm{LF} d \mathrm{~T}$ profiles of all the subjects, yielding values lower than 0.5 in most cases. This is in agreement with the results reported by Rizas et $\mathrm{al},{ }^{13}$ who showed that LF oscillations in ventricular repolarization were also independent of $\mathrm{HRV}$, as assessed through fixed atrial pacing. In Figure 2A-C, different behavior of ventricular repolarization instability and of HRV during exercise can be noticed. Moreover, mechanical effects of respiration are not thought to have a meaningful effect on our repolarization profile analysis, since LF oscillations of repolarization instability have been reported to be unrelated to respiratory activity ${ }^{13}$ and, in the studied scenario, respiratory rate is not expected to lie within the LF band. Additionally, we analyzed the possibility that the increased repolarization instability after reaching VT2 might be due to the effect of cardiolocomotor coupling. ${ }^{18}$ However, the analysis of the spectral alias due to the pedaling cadence did not reveal an influence of cardiolocomotor coupling on ventricular repolarization dynamics (see Figure 2D).

Our results lead to the following interpretation of the $d \mathrm{~T}$ and LF $d \mathrm{~T}$ profiles in the analyzed exercise recordings:

1. Repolarization instability, quantified from $d \mathrm{~T}$ and its LF oscillations $L F d T$, remains low at rest, and it presents a rapid increase following the onset of exercise. This is accompanied by a sudden drop in HRV (see Figure $2 \mathrm{C}$ ), as reported in previous works. ${ }^{9,30}$

2. Afterward, the repolarization dynamics present a plateaulike behavior, which might be due to the fact that, during light-intensity exercise, vagal withdrawal is the main (even if not the only) autonomic modulator. ${ }^{30}$ The sparse cholinergic innervation of human ventricles ${ }^{31}$ could explain why a reduction in repolarization instability is not observed.

3. Cardiac parasympathetic drive reduces and keeps low until 50-60\% of $\dot{\mathrm{V}}_{\mathrm{O}_{2, \max }}{ }^{32,33}$ (see Figure 2C). In this isocapnic buffering stage, the progressive increase in blood lactate levels is compensated with the $\mathrm{HCO}_{3}^{-}$buffer system. The increase in $\mathrm{LF} d \mathrm{~T}$ after this point (see Figure $2 \mathrm{~B}$ ) might be reflecting an increased sympathetic modulation of ventricular electrical function. ${ }^{30,34}$

4. Upon reaching VT2, the increase in blood lactate cannot be further compensated. VT2 is called threshold of decompensated metabolic acidosis: since, at high intensities, the hyperpnea is not sufficient to eliminate the metabolic production of $\mathrm{CO}_{2}, \dot{\mathrm{V}}_{\mathrm{E}}$ increases whereas $\dot{\mathrm{V}}_{\mathrm{CO}_{2}}$ remains constant, leading to a drastic increase in $\dot{\mathrm{V}}_{\mathrm{E}} / \dot{\mathrm{V}}_{\mathrm{CO}_{2}}$ until exhaustion. As an accompaniment, there is a sympathetic-mediated increase in the respiratory rate aiming at compensating metabolic acidosis by respiratory alkalosis ( $\mathrm{CO}_{2}$ elimination). It is possible that this effect is reflected as a new increase in repolarization instability (see Figure 2A,B), since blood catecholamines levels increase exponentially after reaching VT2. ${ }^{35}$ In our case, VT2 was in mean located at $80 \%$ of $\dot{\mathrm{V}}_{\mathrm{O}_{2, \max }}$ (see Table 2), in accordance with previous studies reporting that trained subjects reach VT2 at around 70\%-90\% of their $\dot{\mathrm{V}}_{\mathrm{O}_{2, \max }}{ }^{36,37}$ On the other hand, in Figure 2C), a rise in $\mathrm{P}_{\mathrm{HF}}$ can be also observed, although the increase of the HF component of HRV at high exercise intensities has been related to mechanical effects of respiration. ${ }^{11}$

5. Finally, the reduction in repolarization instability following the end of exercise would be associated with a decrease in sympathetic activity and a restitution of sympathovagal control. 
The estimation of AnT from the analysis of ventricular repolarization dynamics was recently proposed by Hamm et al, ${ }^{12}$ who studied the moving correlation between the instantaneous HR and the $d$ T profile. The time point at which this correlation was minimum was used as an estimate of $\mathrm{AnT}_{\mathrm{LA}}$. In a first analysis, we applied the methodology described in ${ }^{12}$ to the exercise recordings of this study. However, large estimation errors were obtained due to the highly heterogeneous correlation profiles for the different analyzed subjects. In view of those results, we developed a methodology that could be better suited for the analysis of our exercise recordings. The low performance of the methods proposed in ${ }^{12}$ can at least be partially explained based on relevant differences between our study and the one by Hamm et al. First, the design of the exercise test was very different, being it much more exigent in our case, with an initial workload of 75 $\mathrm{W}$ followed by increments of $25 \mathrm{~W}$ per minute, in comparison with, ${ }^{12}$ with an initial workload of $40 \mathrm{~W}$ and increases of $30 \mathrm{~W}$ every 3 minutes. This results in different HR profiles, with a much smoother increase in the case of,${ }^{12}$ which could in turn justify the observed differences in the correlation profiles.

Another important difference between our study and the study by Hamm et al. lies on the lead configuration of the ECG acquisition. Whereas in ${ }^{12}$ the orthogonal Frank's leads were employed, in our case the conventional 12-lead ECG configuration was employed, except for leads V1, V2, and V3, which were not available because the volunteers were also wearing a chest band during the exercise test. The absence of lead V2 made it impossible to have three orthogonal leads, so a quasi-orthogonal lead configuration composed of V4, V6, and aVF was employed. However, due to the low quality of lead aVF in several subjects, a method based on the use of only leads V4 and V6 was proposed. As shown in Figure 4 for cases where aVF was of good quality, the effect of using two leads instead of three resulted in very small differences for most of the subjects in the case of $\mathrm{VT} 2_{d \mathrm{~T}}$, whereas it had greater impact in $\mathrm{VT}_{\mathrm{LF} d \mathrm{~T}}$, with errors being larger when employing the three-lead configuration. In this way, the availability of a lower amount of information in the case of using two leads only does not essentially alter the trend of $d \mathrm{~T}$, possibly due to the fact that the repolarization vector of the $\mathrm{T}$ waves is mainly contained in the horizontal plane. $^{38}$

Nevertheless, it is important to highlight that the ECG data used in this study were acquired using a Holter recorder designed for medical/clinical applications. Currently, specific Holter recorders for sport applications are being designed, which could allow acquiring ECG signals of higher quality. Moreover, other commercial devices already allow registering an ECG lead (eg, Polar H10), being referred to as a gold standard for HR monitoring during exercise, for example, jogging on a treadmill at $11 \mathrm{~km} / \mathrm{h} .{ }^{39}$ When these devices are technologically developed to include a second lead, they could be used for VT2 estimation from $d \mathrm{~T}$ according to our proposed method.

A third relevant difference between our study and the study by Hamm et al. refers to the fact that the gold standard in $^{12}$ was $\mathrm{AnT}_{\mathrm{LA}}$, while in this work VT2 was employed. In spite of the fact that $\mathrm{AnT}_{\mathrm{LA}}$ is largely acknowledged as a good estimator of the physical condition, there is still not a consensus on its use as an AnT estimate, since its accuracy is highly dependent on the procedure and site of blood extraction, as well as on the methods employed for its analysis. ${ }^{40}$ Moreover, the estimates obtained using $\mathrm{AnT}_{\mathrm{LA}}$ or VT2 as gold standard are dependent on the sampling period. $\operatorname{In}^{12}$ the sampling period was selected to be 3 minutes, which is much larger than in the case of the VT2 analyzed in this study, where the signals used for its determination have a more continuous-time nature. Nevertheless, weaknesses in the determination of VT2 must be acknowledged, as this can be subjective in some cases, depending on the expert annotator experience and performance, or on the established criteria in the case of automatic detection.

Other non-invasive approaches for AnT estimation have been proposed in the literature. Assessment of EMG activity has been also recursively employed for VT2 estimation. ${ }^{6,7}$ Even if some EMG-based approaches render low estimation errors, the use of wearables for EMG recording is far less extended than for cardiac activity recording, which limits their application. On the other hand, the use of HRV has been shown to render high performance. ${ }^{10}$ However, the authors employed SD1 calculated from the Poincaré plot of the HRV series, which is mostly related to HF modulation of HR. Given that mechanical effect of respiration is thought to be the main HF modulator of HR at high-intensity exercise,${ }^{11}$ it is possible that the estimates in ${ }^{10}$ are not reflecting a change in autonomic regulation in the proximity of VT2, thus confounding its physiological interpretation.

Several limitations of this work are acknowledged. (1) The use of only two leads for VT2 estimation limits the amount of information on ventricular repolarization dynamics. Nevertheless, the addition of a third orthogonal lead only remarkably improves VT2 estimation in half of the recordings in the case of $\mathrm{VT} 2_{\mathrm{LF} d \mathrm{~T}}$, while in the case of $\mathrm{VT} 2_{d \mathrm{~T}}$ the results do not change considerably. (2) The use of predefined HR thresholds in our methods could result in VT2 overestimation. However, in this study, none of the subjects had reached their VT2 at the established threshold of $77 \%$ of their maximum HR. (3) The reference VT2 was assessed by a single evaluator, which might constitute an additional source of error. However, the achieved estimation error was lower than the inter-evaluator error reported in previous studies, as discussed above. (4) The reduced number of subjects in the dataset, together with the narrow interval of age and physical conditions of the participants (all of them were used to 
aerobic training) and the fact that the study was performed under laboratory conditions, make further studies necessary to validate the described methodology. In particular, application of the methodology to people with limited exercise capacity would be of interest. (5) Finally, it is important to highlight that good ECG signal quality is required for proper delineation of the $\mathrm{T}$ waves. Based on this criterion, $20 \%$ of the recordings had to be discarded. Although this limitation might be overcome in a controlled environment, it is important to perform further validation in additional scenarios, such as treadmill tests. For those future studies, we plan to develop novel strategies for $\mathrm{T}$ wave analysis not requiring full delineation of the $\mathrm{T}$ wave.

Nonetheless, the non-invasive nature of our proposed methods allows estimating VT2 by using only two ECG leads which could be integrated in a chest band, thus resulting in a high ecological validity, especially in outdoor sports such as mountain biking, canoeing, swimming, or cross-country skiing. Moreover, such a non-invasive approach could be useful for obtaining large data samples, aiding in the characterization of VT2 in the general population.

\section{5 | PERSPECTIVE}

A novel methodology for VT2 estimation based on the analysis of ventricular repolarization during exercise has been proposed. This method renders high performance, improving previous results based on similar approaches. As compared to VT2 estimation from gas exchange, our method does not require specific equipment or qualified personnel. Moreover, it is objective, with the achieved mean estimation error being lower than inter-evaluator agreement based on traditional methods.

Differently from the method proposed by Hamm et al ${ }^{12}$ only two precordial leads are required by our method. Therefore, it could be implemented in wearable devices for monitoring cardiac activity, thus allowing to characterize VT2 in the general population, with a high ecological validity.

Importantly, we also proposed a hypothesis for the physiological mechanisms underlying the repolarization patterns during exercise on which our method is based.

Nevertheless, good ECG signal quality is required for our method to carry out the estimation. Future studies are aimed at extending the method to signals with lower signal-to-noise ratio. We only validated our method in a reduced group of subjects of similar age and physical condition, all cases performing a cycle ergometer test. Further work is needed to test the proposed methodology over other populations.

\section{'AUTHOR CONTRIBUTION}

All authors equally contributed to the conception of the work, revising it critically for important intellectual content, final approval of the version to be published, and to the discussion and interpretation of the results. Additionally, Bailón and Pueyo supervised this work, also giving methodological support. Hernando prepared the datasets for the analysis. Hernández-Vicente, Casajús, and Garatachea contributed with methodological and physiological interpretation support. JM was responsible for drafting this work.

\section{ACKNOWLEDGEMENTS}

This work was supported by grant BES-2015-073694 and by projects RTI2018-097723-B-I00 and PID2019105674RB-I00 from Ministerio de Economía y Competitividad and Ministerio de Ciencia e Innovación. Also by the European Research Council through grant ERCStG 638284, by Gobierno de Aragón (Reference Group BSICoS T39-20R and projects LMP124-18 and LMP44_18) cofounded by FEDER 2014-2020 "Building Europe from Aragón," by CIBER in Bioengineering, Biomaterials \& Nanomedicine (CIBER-BBN) through Instituto de Salud Carlos III, and by Ministerio de Educación, Cultura y Deporte (grant number FPU16 / 05879) to AH. The computation was performed by the ICTS NANBIOSIS, specifically by the High Performance Computing Unit of CIBER-BBN at University of Zaragoza.

\section{CONFLICT OF INTEREST}

The authors declare that there is no conflict of interest regarding the publication of this paper.

\section{ORCID}

Javier Milagro (iD https://orcid.org/0000-0002-7842-9611

\section{REFERENCES}

1. Porcari J, Bryant C, Comana F. Exercise Physiology. Philadelphia, PA: FA Davis; 2015.

2. Wasserman K. The anaerobic threshold: definition, physiological significance and identification. Adv Cardiol. 1986;35:1-23.

3. Meyer T, Lucía A, Earnest CP, Kindermann W. A conceptual framework for performance diagnosis and training prescription from submaximal gas exchange parameters-theory and application. Int J Sports Med. 2005;26:38-48.

4. Balady GJ, Arena R, Sietsema K, et al. Clinician's guide to cardiopulmonary exercise testing in adults: a scientific statement from the American Heart Association. Circulation. 2010;122:191-225.

5. Svedahl K, MacIntosh BR. Anaerobic threshold: the concept and methods of measurement. Can J Appl Physiol. 2003;28:299-323.

6. Tikkanen $\mathrm{O}, \mathrm{Hu} \mathrm{M}$, Vilavuo T, et al. Ventilatory threshold during incremental running can be estimated using EMG shorts. Physiol Meas. 2012;33:603.

7. Kang S-K, Kim J, Kwon M, et al. Objectivity and validity of EMG method in estimating anaerobic threshold. Int J Sports Med. 2014;35:737-742.

8. Hofmann P, Bunc V, Leitner H, et al. Heart rate threshold related to lactate turn point and steady-state exercise on a cycle ergometer. Eur J Appl Physiol Occup Physiol. 1994;69:132-139. 
9. Karapetian GK, Engels HJ, Gretebeck RJ. Use of heart rate variability to estimate LT and VT. Int J Sports Med. 2008;29:652-657.

10. Ramos-Campo DJ, Rubio-Arias JA, Ávila-Gandía V, et al. Heart rate variability to assess ventilatory thresholds in professional basketball players. J Sport Health Sci. 2017;6:468-473.

11. Cottin F, Médigue C, Papelier Y. Effect of heavy exercise on spectral baroreflex sensitivity, heart rate, and blood pressure variability in well-trained humans. Am J Physiol Heart Circ Physiol. 2008;295:H1150-H1155.

12. Hamm W, Von stülpnagel L, Rizas KD, et al. Dynamic changes of cardiac repolarization instability during exercise testing. Med Sci Sports Exerc. 2019;51:1517-1522.

13. Rizas KD, Nieminen T, Barthel P, et al. Sympathetic activity-associated periodic repolarization dynamics predict mortality following myocardial infarction. J Clin Invest. 2014;124:1770-1780.

14. Rizas KD, Hamm W, Kääb S, et al. Periodic repolarisation dynamics: a natural probe of the ventricular response to sympathetic activation. Arrhyth Electrophysiol Rev. 2016;5:31.

15. Tanaka H, Monahan KD, Seals DR. Age-predicted maximal heart rate revisited. J Am Coll Cardiol. 2001;37:153-156.

16. Lucía A, Hoyos J, Carvajal A, et al. Heart rate response to professional road cycling: the Tour de France. Int J Sports Med. 1999;20:167-172.

17. Martinez JP, Almeida R, Olmos S, et al. A wavelet-based ECG delineator: evaluation on standard databases. IEEE Trans Biomed Eng. 2004;51:570-581.

18. Hernando D, Hernando A, Casajús JA, et al. Methodological framework for heart rate variability analysis during exercise: application to running and cycling stress testing. Med Biol Eng Comput. 2018;56:781-794.

19. Goldberg L, Elliot DL, Kuehl KS. Assessment of exercise intensity formulas by use of ventilatory threshold. Chest. 1988;94:95-98.

20. Weltman A, Snead D, Seip R, et al. Percentages of maximal heart rate, heart rate reserve and $\mathrm{VO} 2 \mathrm{max}$ for determining endurance training intensity in male runners. Int J Sports Med. 1990;11:218-222.

21. Neder JA, Stein R. A simplified strategy for the estimation of the exercise ventilatory thresholds. Med Sci Sports Exerc. 2006;38:1007.

22. Bailón R, Laouini G, Grao C, et al. The integral pulse frequency modulation model with time-varying threshold: application to heart rate variability analysis during exercise stress testing. IEEE Trans Biomed Eng. 2010;58:642-652.

23. Task force of the European Society of cardiology and the north american society of pacing physiology. Heart rate variability: standards of measurement, physiological interpretation, and clinical use. Circulation. 1996;93:1043-1065.

24. Laguna P, Moody GB, Mark RG. Power spectral density of unevenly sampled data by least-square analysis: performance and application to heart rate signals. IEEE Trans Biomed Eng. 1998;45:698-715.

25. Gladden LB, Yates JW, Stremel RW, et al. Gas exchange and lactate anaerobic thresholds: inter-and intraevaluator agreement. J Appl Physiol. 1985;58:2082-2089.

26. Hanson B, Child N, Van Duijvenboden S, et al. Oscillatory behavior of ventricular action potential duration in heart failure patients at respiratory rate and low frequency. Front Physiol. 2014;5:414.

27. Porter B, van Duijvenboden S, Bishop MJ, et al. Beat-to-beat variability of ventricular action potential duration oscillates at low frequency during sympathetic provocation in humans. Front Physiol. 2018;9:147.

28. Pueyo E, Orini M, Rodríguez JF, et al. Interactive effect of beta-adrenergic stimulation and mechanical stretch on low-frequency oscillations of ventricular action potential duration in humans. $J \mathrm{Mol}$ Cell Cardiol. 2016;97:93-105.

29. Sampedro-Puente DA, Fernandez-Bes J, Porter B, et al. Mechanisms underlying interactions between low-frequency oscillations and beat-to-beat variability of celullar ventricular repolarization in response to sympathetic stimulation: Implications for arrhythmogenesis. Front Physiol. 2019;10:916.

30. White DW, Raven PB. Autonomic neural control of heart rate during dynamic exercise: revisited. J Physiol. 2014;592:2491-2500.

31. Kawano H, Okada R, Yano K. Histological study on the distribution of autonomic nerves in the human heart. Heart Vessels. 2003;18:32-39.

32. Tulppo MP, Makikallio TH, Takala TE, et al. Quantitative beat-tobeat analysis of heart rate dynamics during exercise. Am J Physiol Heart Circ Physiol. 1996;271:H244-H253.

33. Michael S, Graham KS, Davis GM. Cardiac autonomic responses during exercise and post-exercise recovery using heart rate variability and systolic time intervals - a review. Front Physiol. 2017;8:301.

34. Tulppo MP, Mäkikallio TH, Seppänen T, et al. Vagal modulation of heart rate during exercise: effects of age and physical fitness. Am J Physiol Heart Circ Physiol. 1998;274:H424-H429.

35. Lehmann M, Keul J, Huber G, et al. Plasma catecholamines in trained and untrained volunteers during graduated exercise. Int $J$ Sports Med. 1981;2:143-147.

36. Bunc V, Heller J, Leso J, et al. Ventilatory threshold in various groups of highly trained athletes. Int J Sports Med. 1987;8:275-280.

37. Millet GP, Vleck VE, Bentley DJ. Physiological differences between cycling and running. Sports Med. 2009;39:179-206.

38. Mohrman DE, Heller LJ. Cardiovascular Physiology, 9th edn. McGraw Hill Education; 2018.

39. Gilgen-Ammann R, Schweizer T, Wyss T. RR interval signal quality of a heart rate monitor and an ECG Holter at rest and during exercise. Eur J Appl Physiol. 2019;119:1525-1532.

40. Faude O, Kindermann W, Meyer T. Lactate threshold concepts. Sports Med. 2009;39:469-490.

How to cite this article: Milagro J, HernándezVicente A, Hernando D, et al. Estimation of the second ventilatory threshold through ventricular repolarization profile analysis. Scand J Med Sci Sport. 2020;00:1-11. https://doi.org/10.1111/sms.13849 